\title{
miR-216a-5p inhibits malignant progression in small cell lung cancer: involvement of the Bcl-2 family proteins
}

This article was published in the following Dove Press journal: Cancer Management and Research

\author{
Yanqin Sun ${ }^{1, *}$ \\ Bingshuang $\mathrm{Hu}^{2, *}$ \\ Yanhong Wang ${ }^{3}$ \\ Zhen $\mathrm{Li}^{\prime}$ \\ jingfang $\mathrm{Wu}^{4}$ \\ Yunchu Yang ${ }^{4}$ \\ Yue Wei ${ }^{5}$ \\ Xiaofeng Peng ${ }^{5}$ \\ Hongling Chen ${ }^{5}$ \\ Rongqi Chen ${ }^{5}$ \\ Pingyan Jiang ${ }^{5}$ \\ Sixian Fang ${ }^{5}$ \\ Zhiwu $\mathrm{Yu}^{6}$ \\ Linlang Guo ${ }^{4}$
}

'Department of Pathology, Guangdong Medical University, Dongguan,

China; ${ }^{2}$ Department of Radiotherapy,

Zhongshan People's Hospital,

Zhongshan, China; ${ }^{3}$ Department

of Gynecology, Jinxiang People's

Hospital, Jining, China; ${ }^{4}$ Department of

Pathology, Zhujiang Hospital, Southern

Medical University, Guangzhou, China;

${ }^{5}$ College of Pharmacy, Guangdong

Medical University, Dongguan, China;

${ }^{6}$ Division of Laboratory Science,

Affiliated Cancer Hospital and Institute of Guangzhou Medical

University, Guangzhou, China

*These authors contributed equally to this work

Correspondence: Linlang Guo Department of Pathology, Zhujiang Hospital, Southern Medical University, No 253 Gongye Road, Guangzhou 5I 0282, China

Tel +86206278 3358

Fax +86 2084311872

Email linlangg@yahoo.com
Objective: microRNAs are regulatory molecules regarded as important in the pathogenesis of different types of tumors. microRNA-216a (miR-216a-5p) has been identified as a tumor suppressor in multiple malignancies. However, the role of miR-216a-5p in the pathogenesis of small cell lung cancer (SCLC) remains obscure. The objective of this study was to investigate the role of the miR-216a-5p/Bcl-2 axis in SCLC pathogenesis.

Materials and methods: All the experimental methods used were as follows: microarray analysis, cell culture, transient, and stable gene transfection; real-time fluorescence PCR; Western blot; flow cytometry for cell cycle analysis; in vitro proliferation assay; in vitro wound healing experiment; in vivo xenograft model in nude mice; and dual luciferase reporter assay. All statistical analyses were carried out using GraphPad Prism 7 software. Statistical significance was analyzed by Student's $t$-test or one-way ANOVA. $P<0.05$ (typically compared with the negative control group) was considered as significant and is marked with an asterisk in the figures. Results: In this study, we observed that miR-216a-5p is downregulated in SCLC cell lines compared to that in the normal human bronchial epithelial cell line 16-HBE. In vitro and in vivo experiments demonstrate that upregulation of miR-216a-5p significantly decreased cell growth and migration and its downregulation increased SCLC cell proliferation and migration and influenced the cell cycle. Using bioinformatics analyses, we predicted that the important antiapoptotic gene $B c l-2$ is targeted by miR-216a-5p, and we identified a functional miR216a-5p binding site in the $3^{\prime}$-UTR of Bcl-2 using luciferase reporter assay. Furthermore, we determined that suppression of miR-216a-5p modulated the expression of $B c l-2$, Bax, and Bad proteins ( $B c l-2$ family proteins), while $B c l-2$ knockdown abrogated the effect of miR-216a-5p downregulation on cell proliferation, cell migration, and the cell cycle.

Conclusion: Taken together, these findings suggest that miR-216a-5p regulates SCLC biology via $B c l-2$ family proteins. Therefore, our study highlights the role of the miR-216a-5p/Bcl-2 axis in SCLC pathogenesis.

Keywords: pathogenesis, miR-216a-5p, Bcl-2, SCLC

\section{Introduction}

Small cell lung cancer (SCLC) accounts for $\sim 15 \%$ of all lung cancer cases. ${ }^{1}$ SCLC tumors generally become chemoresistant after effective chemotherapy treatments, and most patients die from a recurrence of cancer or from serious complications. ${ }^{2}$ Hence, it is important to explore the molecular mechanisms underlying SCLC progression. microRNAs (miRNAs), a type of small noncoding RNA with a length of 19-25 nucleotides, can inhibit the expression of their target genes by binding to their $3^{\prime}$-UTR, resulting in mRNA degradation or posttranslational repression. ${ }^{3}$ miRNAs are involved in the differentiation 
of normal tissues and play an important role in the pathogenesis of many human cancers. ${ }^{3}$ Some previous studies have demonstrated that miRNAs are deregulated in SCLC progression. ${ }^{1,4-6}$ According to our previous findings, miR-216a-5p was identified among 24 miRNAs that were abnormally expressed in SCLC H69/H69AR cells based on TaqMan miRNA microarray analysis. ${ }^{7,8} \mathrm{~A}$ number of studies have demonstrated that miR-216a-5p is downregulated in gastric cancer, pancreatic cancer, and colorectal cancer and acts as a tumor suppressor. ${ }^{9-12}$ Therefore, experiments to determine whether miR-216a-5p acts as an oncogene or a tumor suppressor in SCLC are warranted.

\section{Materials and methods Microarray analysis}

For miRNA microarrays (LC Sciences, Houston, TX, USA), differentially expressed miRNAs with a fourfold decrease in the raw expression level were ordered by $P$-value. The experimental procedures were performed as previously described in detail. ${ }^{7}$

\section{Cell culture}

The human SCLC cell lines NCI-H69, NCI-H69AR, NCI$\mathrm{H} 446$, and 16-HBE cells were all from the American Type Culture Collection (ATCC; Manassas, VA, USA), maintained at $37^{\circ} \mathrm{C}$ in a $5 \% \mathrm{CO}_{2}$ humidified incubator and grown in Roswell Park Memorial Institute (RPMI)-1640 medium (Thermo Fisher Scientific, Waltham, MA, USA) supplemented with 10\% FBS (HyClone, Logan, UT, USA). The H69AR cell line is a multidrug-resistant cell line that is primarily used in microarrays to screen for chemoresistance-related miRNAs in SCLC and is occasionally used in this study. ${ }^{7}$

\section{Transient and stable transfection}

The siRNAs, miR-216a-5p mimics, inhibitors, and their respective negative control (NC) vectors were purchased from GenePharma (Shanghai, China) and then transiently and stably transfected according to the manufacturer's instructions. The most effective interference sequences within $B c l-2$ were identified based on real-time quantitative PCR (RT-qPCR) and then used for subsequent in vitro experiments. The stable overexpression or silence miR-216a-5p expression cell lines and control cell lines were constructed by lentivirus infection. The detailed procedure is provided in the "Supplementary materials" section. The relevant mimic and inhibitor sequences are listed in the "Supplementary materials" section.

\section{RT-qPCR}

RT-qPCR was used to detect the expression level of miR-216a-5p and genes in SCLC cells according to the manufacturer's instructions (Takara, Dalian, China). GAPDH was used as the control. The relevant primers are listed in the "Supplementary materials" section.

\section{Western blot analysis}

Western blot analysis was performed according to standard protocols as previously described. ${ }^{13} \mathrm{GAPDH}$ and $\beta$-actin were used as the controls. The antibody information for apoptosisrelated genes is listed in the "Supplementary materials" section.

\section{Flow cytometry for cell cycle analysis}

Cell cycle assays were performed after cells were fixed in $70 \%$ ethanol overnight at $4^{\circ} \mathrm{C}$ and then stained with propidium iodide. The procedure was conducted according to standard protocols described in detail previously. ${ }^{15}$

\section{In vitro proliferation assay}

The Cell Counting Kit-8 (CCK-8; Dojindo, Kyushu Island, Japan) was used for the in vitro proliferation assays in accordance with the manufacturer's instructions as described previously. ${ }^{14,15}$

\section{In vitro wound healing experiment}

SCLC cells were grown to confluence on 24-well plates. Consistently shaped wounds were made using a sterile 10 $\mu \mathrm{L}$ pipette tip. At least four images of the scraped area were captured using phase contrast microscopy immediately after the scratch and after $0,12,24,36,48$, and 72 hours. The procedure was replicated three times and was performed as previously described. ${ }^{16}$ The same scratched area was selected for measurement at each time point.

\section{In vivo xenograft model in nude mice}

Six-to-seven-week-old female BALB/c mice (Guangdong Experimental Animal Center, Guangzhou, China) were accommodated (license number for laboratory animals: SYXY (YUE)2013-0002) and handled in compliance with the current European, national, and local (RD 1201/2005) regulations, following the Federation for Laboratory Animal Science Associations and Animal Research: Reporting of In Vivo Experiments guidelines and with the approval of the Institutional Animal Care Use Committee of Sun Yat-Sen University.

The xenograft tumor formation experiment was conducted according to our standard protocol as described previously. ${ }^{16}$ Animals were randomly divided into four groups ( $\mathrm{n}=3$ mice per group): 1) H69AR/LV-miR-NC; 2) H69/LV-miR-216a-5p inhibitors; 3) H69AR/LV-miR-216a-5p mimics; and 4) H69/ LV-miR-NC. When the average longest tumor diameter of any group reached $\sim 1 \mathrm{~cm}$, the mice were sacrificed, and the excised tumors were measured. 


\section{Dual luciferase reporter assay}

H69 cells were transfected with hsa-miR-216a-5p mimics/ inhibitors/NC by Lipofectamine 2000 (Thermo Fisher Scientific). Dual Luciferase Reporter Assay System (Promega, Madison, WI, USA) was used to check the luciferase activity of H69 cell lysates. The detailed procedure was conducted according to our standard protocol as described previously. ${ }^{17}$ All treatments were performed in triplicate.

\section{Statistical analyses}

All experiments were conducted in triplicate. The data are expressed as mean $\pm \mathrm{SD}$. All statistical analyses were carried out using GraphPad Prism 7 software. Statistical significance was analyzed by Student's $t$-test or one-way ANOVA. $P<0.05$ (typically compared with the NC group) was considered as significant and is marked with an asterisk in the figures.

\section{Results \\ Expression of miR-216a-5p was determined in SCLC cell lines}

To identify miRNAs differentially expressed in SCLC, we performed TaqMan miRNA microarray analysis on the H69 and H69AR cell lines (ATCC; Supplementary materials). In all, 24 miRNAs with statistically significant differences in expression of more than fourfold were identified (Figure 1A and Table 1). Our previous studies have demonstrated that Bcl-2 had been proved to be involved in the development of SCLC, even in the occurrence of drug resistance. ${ }^{18}$ We also found that $\mathrm{Bcl}-2$ contains putative regions that match to the seed sequence of miR-216a-5p but not to other 23 miRNAs by further searching in RNA22-seq database (data not shown). Therefore, miR-216a-5p was chosen as an miRNA of interest for further study. By further verification with RT-qPCR, miR-216a-5p showed a significant downregulation in SCLC cell lines compared with that in the normal human bronchial epithelial cell line 16-HBE (ATCC), and the expression of miR-216a-5p in H69 cells was higher than that in H446 cells (ATCC; Figure 1B). Accordingly, the H69 cell line, which exhibits a high level of expression of miR-216a-5p, was used for loss-of-function experiments, and the $\mathrm{H} 446$ cell line was used for gain-of-function experiments.

In order to manipulate miR-216a-5p levels in SCLC cells, miR-216a-5p mimics and inhibitors (GenePharma) were transfected into H446 and H69 cells, respectively. RTqPCR analysis of miR-216a-5p expression was performed at 24 hours after transfection, and the results revealed that the miR-216a-5p level was effectively increased or decreased. The observed inhibition level of miR-216a-5p expression caused by miR-216a-5p inhibitor treatment was $79.7 \%$, and its level of overexpression caused by miR-216a-5p mimic treatment was $168.04 \%$ (Figure 1C). The miR-216a-5p mimics and inhibitors were used in subsequent loss- and gain-of-function studies. For stable transfection, the mimics and inhibitors were packaged into a lentiviral vector for the subsequent studies (Figure 1D), and analysis demonstrated that their ability to increase or decrease expression of miR216a-5p was significant (Figure 1E).

\section{miR-2 I6a-5p regulates SCLC cell growth in vitro and in vivo}

To investigate the effect of miR-216a-5p on the pathogenesis of SCLC in vitro, a CCK-8 assay was carried out in miR216a-5p up- and downregulated cells. The CCK- 8 assay revealed that downregulation of miR-216a-5p increased H69 cell proliferation compared with that in the $\mathrm{NC}$ group, while upregulation of miR-216a-5p decreased cell proliferation (Figure 2A).

The ability of miR-216a-5p to confer SCLC characteristics was examined using an in vivo xenograft model in nude mice. As shown in Figure 2B, tumor growth in mice was significantly increased in the H69/LV-miR-216a-5p inhibitors group compared to that in the H69/LV-miR-NC group and was significantly decreased in the H69AR/LV-miR-216a-5p mimics group compared to that in the H69AR/LV-miR-NC group. At 21 days after subcutaneous injection, the mean tumor volume for the H69/LV-miR-216a-5p inhibitors group was markedly larger than that for the H69/LV-miR-NC group and that for the H69AR/LV-miR-216a-5p mimics group was smaller compared to that of the H69AR/LV-miR-NC group (Figure 2C).

\section{miR-2 I 6a-5p suppression resulted in cell cycle disorder}

To further investigate the growth promotion observed in miR-216a-5p downregulated cells, cell cycle profiles of miR-216a-5p downregulated cells were evaluated using flow cytometry. The suppression of miR-216a-5p led to cell blockade characterized by $\mathrm{G} 2 / \mathrm{M}$ phase arrest and an increase in the number of SCLC cells in the G2/M phase (Figure 2D).

\section{miR-2 I 6a-5p promotes cell migration in vitro}

A scratch healing test was used to determine the effect of miR-216a-5p on cell migration. The results showed that miR-216a-5p upregulation led to a significant reduction in the wound closure rate in H446 cells (Figure 2E and F). 
A

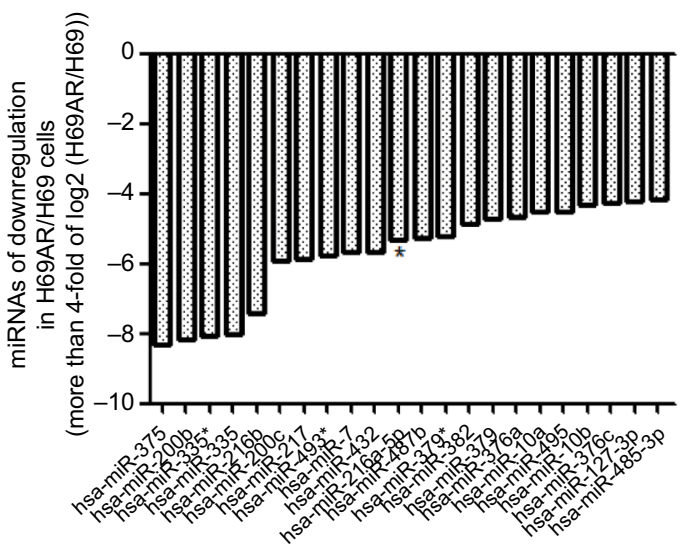

C

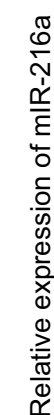

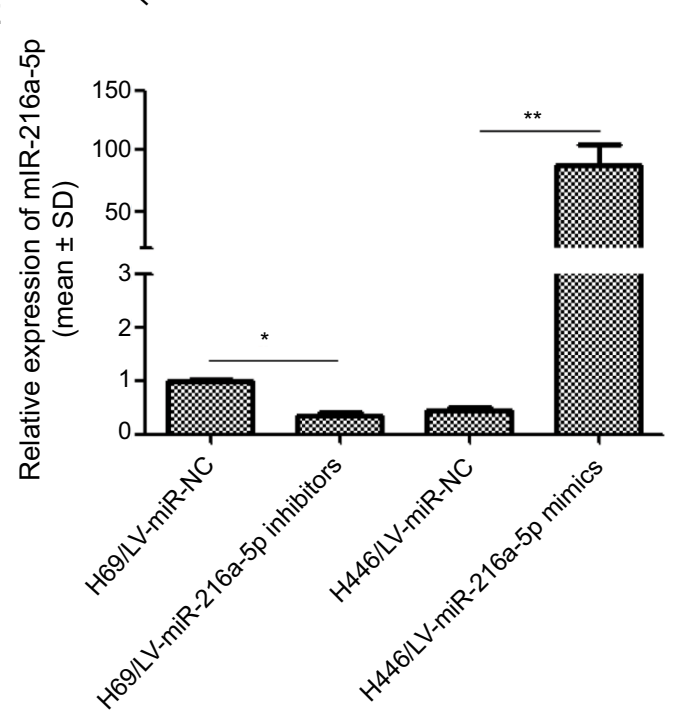

B

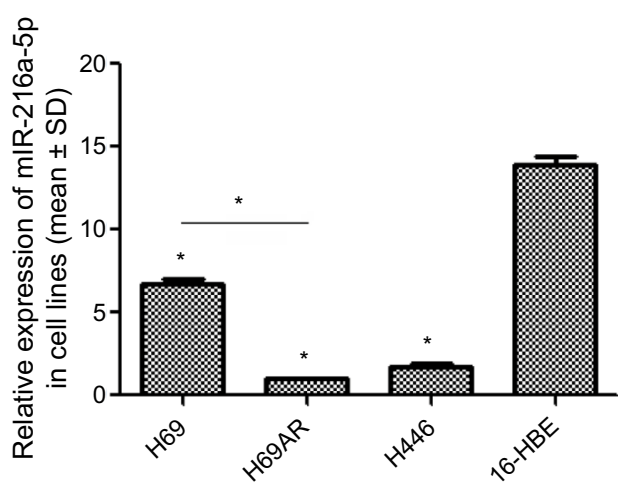

D
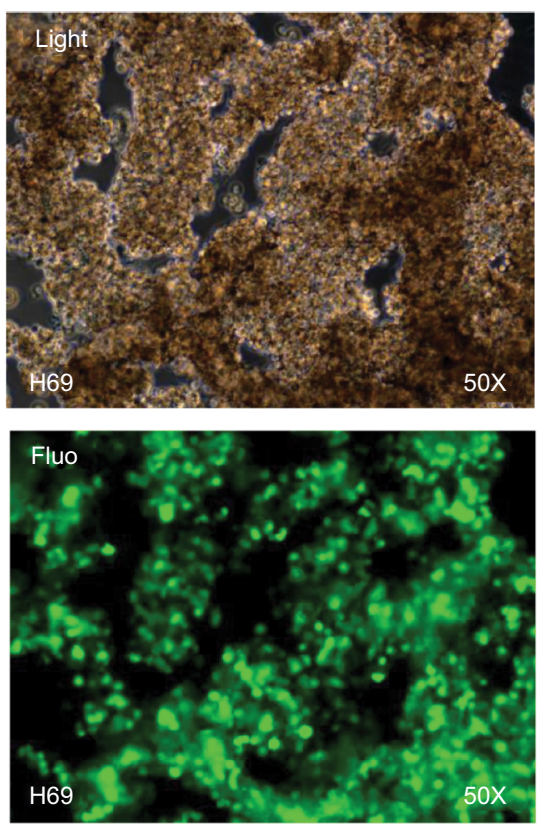

Figure I Relative expression of miR-216a-5p in cell lines.

Notes: (A) Differentially expressed transcripts of miRNAs are provided following the chip images. (B) Expression level of miR-2I6a-5p in three SCLC cell lines and the 16-HBE cell line shows downregulation in SCLC cell lines, especially in the H69AR cell line, compared with that in the 16-HBE cell line. (C) Identification of miR-216a-5p upregulation in $\mathrm{H} 69$ cells and downregulation in $\mathrm{H} 446$ cells. For stable miR-216a-5p upregulation and downregulation, the inhibitors and mimics of miR-2I6a-5p were packaged into a lentivirus for subsequent studies. The transfection efficacy was validated by fluorescence microscopy in H69 cells (D) and RT-qPCR in both the cell lines (E). $* P<0.05$ and $* * P<0.001$.

Abbreviations: miRNA, microRNA; SCLC, small cell lung cancer; RT-qPCR, real-time quantitative PCR. 
Table I Partial differential expression of miRNAs in SCLC H69/ H69AR cells

\begin{tabular}{|c|c|c|c|c|}
\hline \multicolumn{2}{|c|}{ No. Probe_ID } & \multirow{2}{*}{$\begin{array}{l}\text { H69-cy3 } \\
\text { Signal } \\
6,454.34\end{array}$} & \multirow{2}{*}{$\begin{array}{l}\text { H69AR-cy3 } \\
\text { Signal } \\
20.05\end{array}$} & \multirow{2}{*}{$\begin{array}{l}\log 2 \\
(\text { H69AR/H69) } \\
-8.31\end{array}$} \\
\hline I & hsa-miR-375 & & & \\
\hline 2 & hsa-miR-200b & $6,273.58$ & 23.22 & -8.19 \\
\hline 3 & hsa-miR-335* & $3,835.09$ & 13.18 & -8.07 \\
\hline 4 & hsa-miR-335 & $3,663.11$ & 13.79 & -8.04 \\
\hline 7 & hsa-miR-2I6b & I,367.48 & 7.65 & -7.41 \\
\hline 8 & hsa-miR-200c & $17,307.45$ & 278.54 & -5.94 \\
\hline 9 & hsa-miR-2I7 & 448.40 & 6.73 & -5.89 \\
\hline 10 & hsa-miR-493* & 468.53 & 9.02 & -5.75 \\
\hline II & hsa-miR-7 & $8,780.53$ & 174.39 & -5.65 \\
\hline 12 & hsa-miR-432 & $2,|| 6.5 \mid$ & 42.31 & -5.65 \\
\hline 13 & hsa-miR-216a-5p & 305.13 & 7.61 & -5.33 \\
\hline 14 & hsa-miR-487b & 593.66 & $|5.5|$ & -5.26 \\
\hline 15 & hsa-miR-379* & 87.59 & 2.99 & -5.22 \\
\hline 16 & hsa-miR-382 & 525.03 & 19.72 & -4.86 \\
\hline 17 & hsa-miR-379 & 421.69 & 16.33 & -4.70 \\
\hline 18 & hsa-miR-376a & 154.50 & 6.80 & -4.66 \\
\hline 19 & hsa-miR-I0a & 166.79 & 7.61 & -4.54 \\
\hline 20 & hsa-miR-495 & 432.48 & 19.53 & $-4.5 I$ \\
\hline 21 & hsa-miR-I0b & 239.12 & 12.19 & -4.34 \\
\hline 22 & hsa-miR-376c & 152.76 & 8.74 & -4.26 \\
\hline 23 & hsa-miR-127-3 p & 264.57 & 14.57 & -4.21 \\
\hline 24 & hsa-miR-485-3 p & 201.48 & 11.46 & -4.15 \\
\hline
\end{tabular}

Note: $* P<0.05$.

Abbreviations: miRNA, microRNA; SCLC, small cell lung cancer.

\section{$\mathrm{Bcl}-2$ is predicted and confirmed to be a miR-2I6a-5p target gene}

Using miRNA target detection software RNA22-seq (https:// cm.jefferson.edu/), miR-216a-5p was predicted to bind to the apoptosis-related gene $B c l-2$ (Figure 3A). The predicted binding relationship was confirmed by RT-qPCR and Western blot in SCLC cells. The results demonstrated that miR-216a-5p acts as an inhibitor of $\mathrm{Bcl}-2$ (Figure 3B and C). Moreover, inhibition of miR-216a-5p expression in H69 and H69AR cells led to a significantly corresponding increase in $\mathrm{Bcl}-2$ expression (Figure 3B and C).

To further investigate the regulatory relationship between miR-216a-5p and $B c l-2$, we constructed $B c l-2$ wild-type and mutant psicheck-2.0 luciferase vectors and conducted a luciferase reporter assay (Figure 3D). Luciferase activity was reduced by $\sim 59 \%$ (Figure $3 \mathrm{E}$ ) when miR-216a-5p was upregulated and was increased by $50.1 \%$ when miR$216 \mathrm{a}-5 \mathrm{p}$ was downregulated. These data demonstrate that miR-216a-5p binds directly to the Bcl-2 3'-UTR through its miRNA binding sites, leading to miRNA-induced repression of luciferase reporter activity.
miR-2 $16 a-5 p$ regulates the expression of apoptosis-related gene $\mathrm{Bcl}-2 / \mathrm{Bax} / \mathrm{Bad}$

To investigate the regulatory effect of miR-216a-5p on its target gene $B c l-2$, RT-qPCR was used to verify that downregulation of miR-216a-5p leads to an increase in $\mathrm{Bcl}-2$ mRNA expression. Additional experiments showed that siRNA-mediated $B c l-2$ knockdown rescued the overexpression of $B c l-2$ caused by miR-216a-5p inhibition (Figure 4A). Hence, a direct relationship between miR-216a-5p and Bcl-2 was confirmed.

To determine whether miR-216a-5p-mediated regulation of the important apoptosis suppressor gene $\mathrm{Bcl}-2$ is incidental, we performed RT-qPCR and Western blot analysis to assess the effect of miR-216a-5p on the expression of the apoptosis-promoting genes Bax and Bad. The results demonstrated that miR-216a-5p downregulation led to a significant decrease in the mRNA (Figure 4B) and protein levels (Figure 4C) of both Bax and Bad. Previous studies indicated that miR-216a-5p promotes the occurrence of apoptosis in SCLC. We speculated that miR-216a-5p participates in the development of SCLC by promoting apoptosis and affecting the apoptosis pathway.

\section{Bcl-2 partly mediates the effect of miR- $216 a-5 p$ on SCLC cell proliferation and migration}

Interestingly, we found that $\mathrm{Bcl}-2$ knockdown also caused a significant inhibition of SCLC cell growth, as measured by CCK-8 assay, just as miR-216a-5p upregulation did (Figure 4D). Moreover, flow cytometry experiment revealed that $B c l-2$ knockdown rescued the impact of miR-216a-5p downregulation on cells arrested in the $\mathrm{G} 2$ phase, but the S-phase does not seem to be impacted by Bcl-2 siRNA (Figure 4E and F). Meanwhile, different from the increase in cell migration caused by miR-216a-5p downregulation, as shown in the wound healing assay, $B c l-2$ knockdown led to a reduction in cell migration ability compared with the $\mathrm{NC}$ treatment (Figure 4G). Taken together, these results further substantiate the presumptive role of miR-216a-5p and $\mathrm{Bcl}-2$ in SCLC cell cycle progression and provide new evidence to support the concept of their interdependent expression.

\section{Discussion}

SCLC is an aggressive malignancy characterized by high incidence, rapid development, and low survival rate..$^{19,20}$ Although aberrant expression of miRNAs in lung cancer has 
A

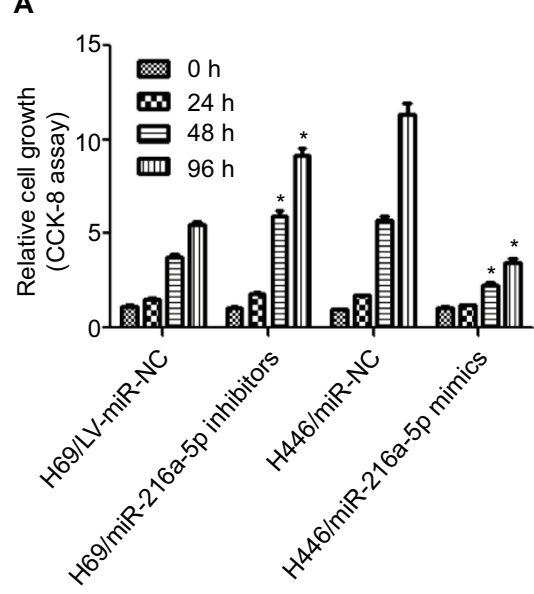

B

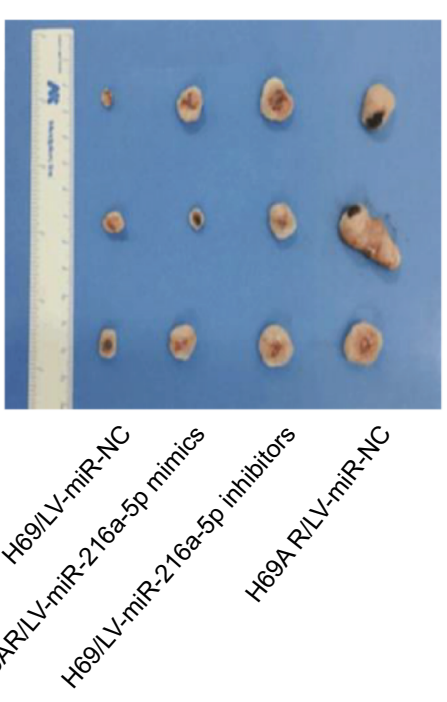

C

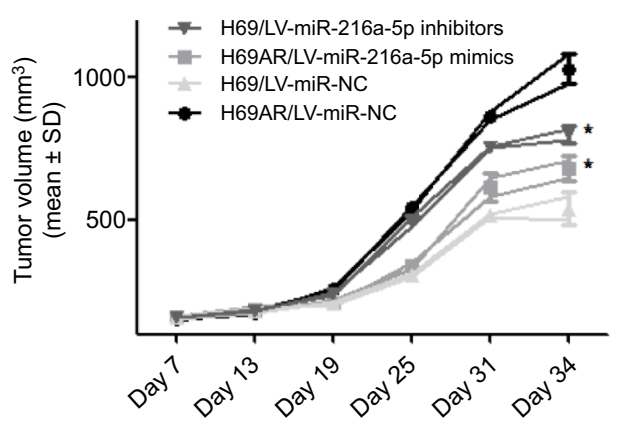

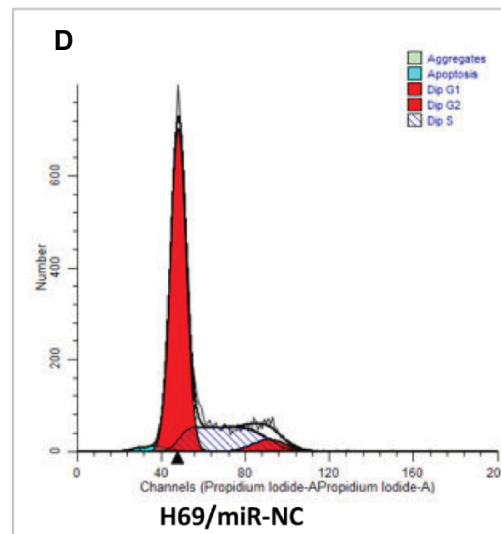

H69/miR-NC

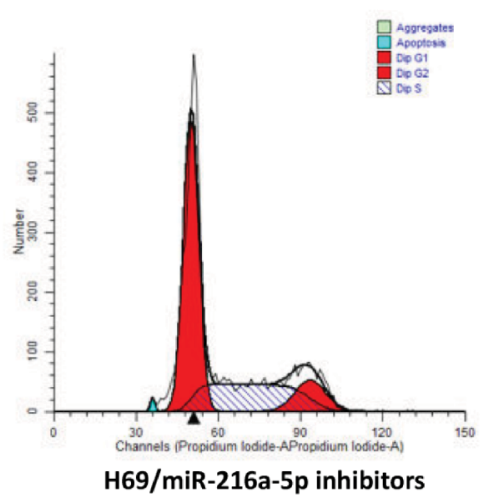

H69

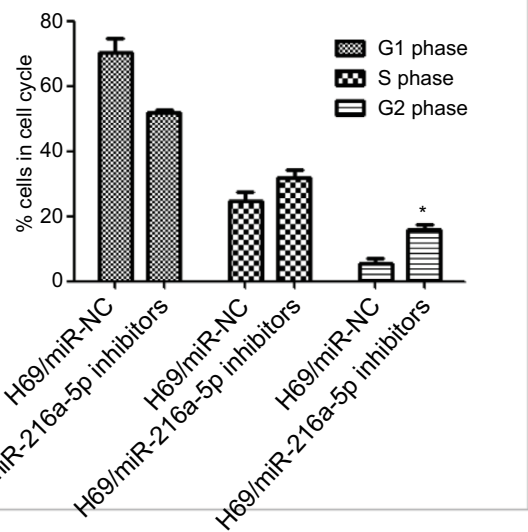

$\mathbf{F}$
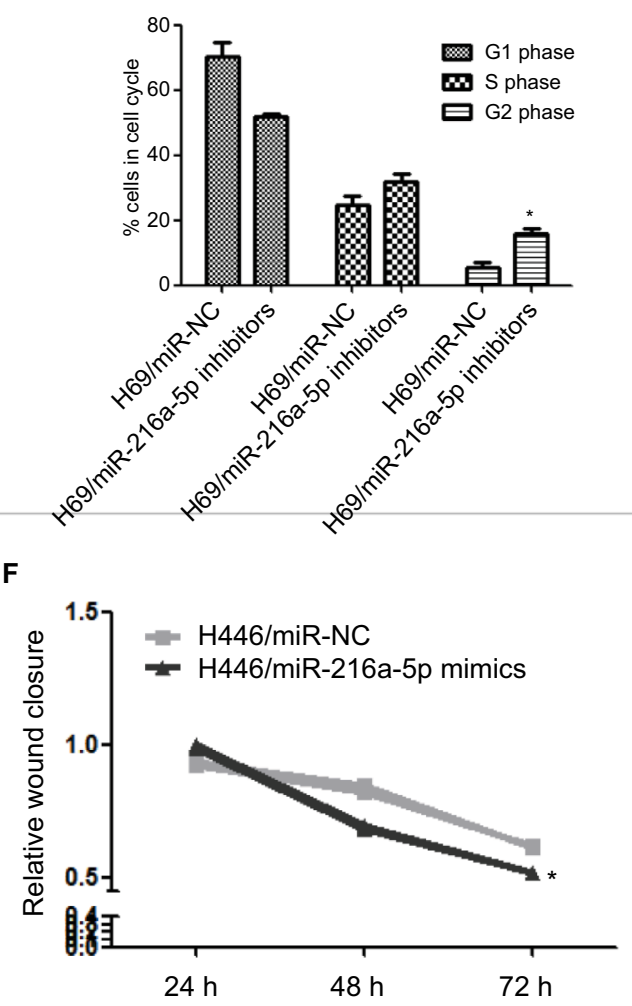

E

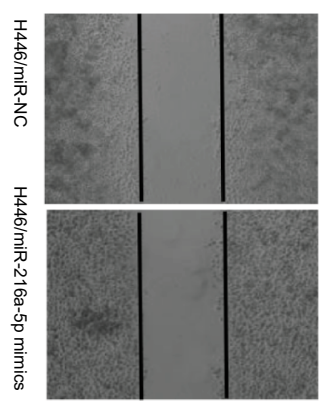

$24 \mathrm{~h}$

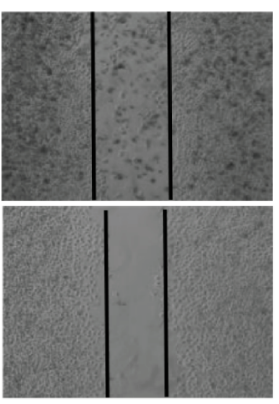

$48 \mathrm{~h}$

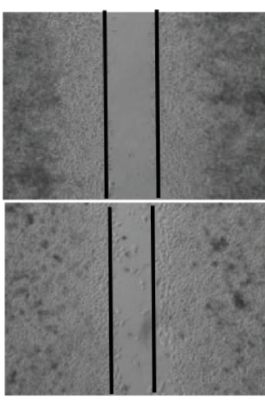

$72 \mathrm{~h}$

ude mice.

Figure 2 miR-216a-5p inhibits SCLC cell proliferation and migration in vitro and decreases growth of xenografts in nude mice.
Notes: (A) A CCK-8 assay was used to determine the effect of miR-216a-5p up- or downregulation on SCLC cell proliferation. (B) Images of excised tumors from tumorbearing nude mice in each group. (C) Tumor volume change for each group measured on the indicated days. (D) Flow cytometric analysis was used for cell cycle phase detection in miR-216a-5p-downregulated H69 cells. (E) miR-216a-5p suppressed SCLC cell migration. (F) Quantitative results of the wound closure rate for miR-2I6a-5pupregulated $\mathrm{H} 446$ cells. $* P<0.05$.

Abbreviations: SCLC, small cell lung cancer; CCK-8, Cell Counting Kit-8; NC, negative control.

been reported, few studies have elucidated the role of specific miRNAs in SCLC tumorigenesis. ${ }^{21}$ In the present study, miRNA microarray analysis identified 24 miRNAs with a significantly downregulated expression in the SCLC chemoresistant H69AR cell line compared with that in the H69 cell line. Therefore, we focused on analyzing miR-216a-5p 
A

\section{3' aguGUCAACGGUCGACUCUAAu 5 ' hsa-miR-216a-5p I $\vdots$ II I $\vdots$ ～I I I I I I \\ 5' gauCGUUUUCUGUUUGAGAUUu 3 , $\mathrm{Bcl}-2$}

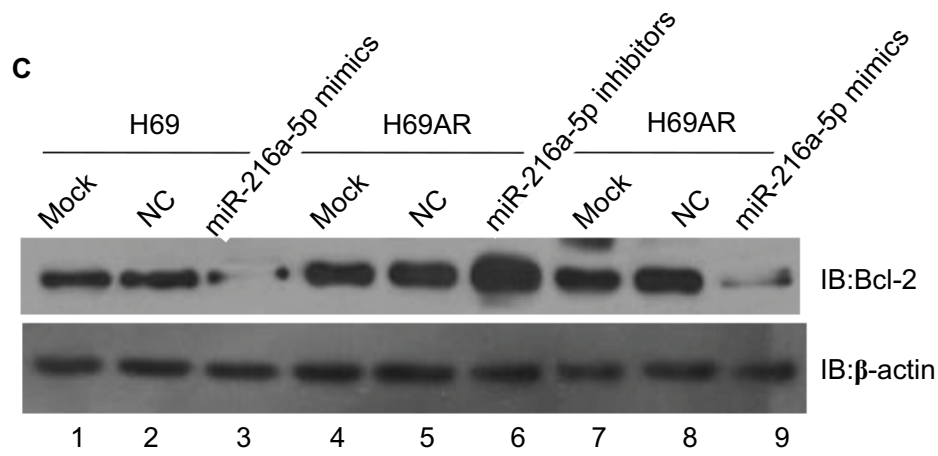

D

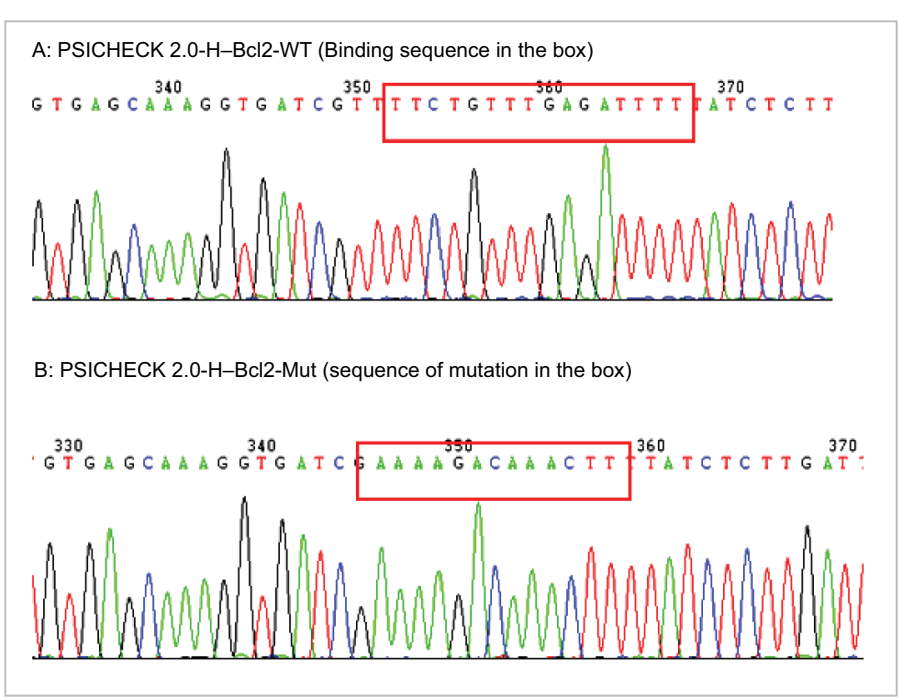

B

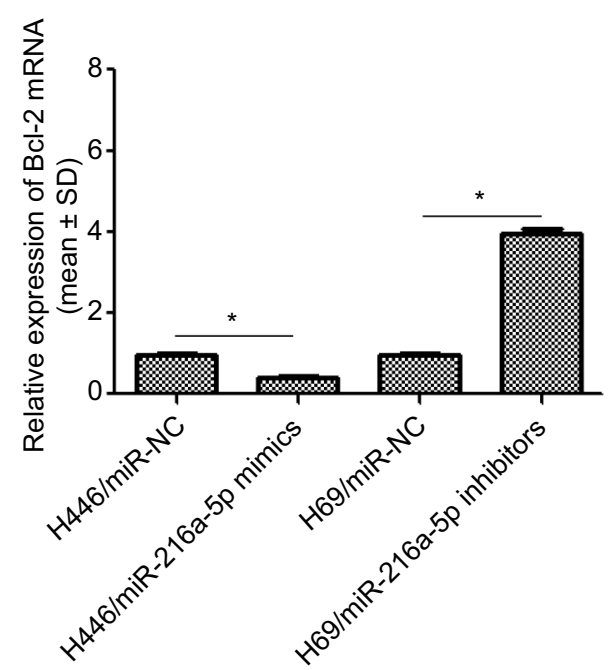

E

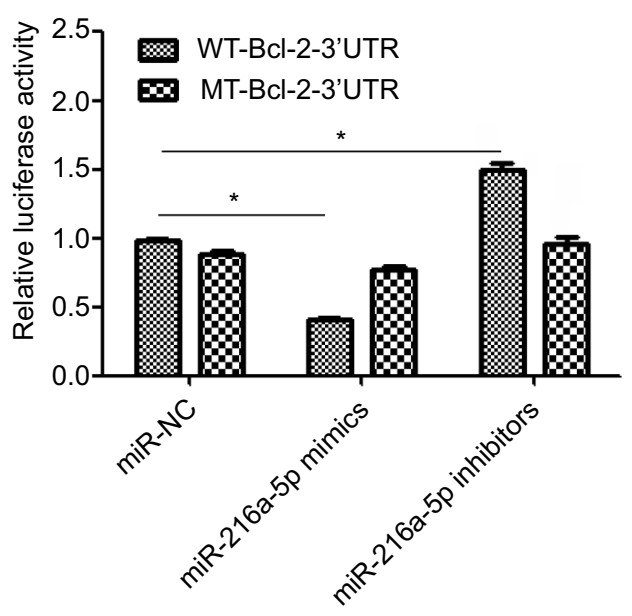

Figure $3 \mathrm{Bcl}-2$ is a target gene of miR-216a-5p.

Notes: (A) Putative binding site of miR-2 I6a-5p in the Bcl-2 3'-UTR and the site of target mutagenesis. (B) miR-216a-5p negatively regulated the expression of its target gene $\mathrm{Bcl}-2$. (C) miR-2 16a-5p negatively regulated the expression of the Bcl-2 protein. (D) Binding and mutation sequences used in the luciferase reporter assay. (E) The luciferase reporter assay was used to demonstrate the target binding relationship between miR-216a-5p and Bcl-2. ${ }^{*} P<0.05$.

Abbreviation: NC, negative control.

in detail. Our previously published microarray study, which screened SCLC lines for chemoresistance-associated miRNAs, ${ }^{7}$ surprisingly found that miR-216a-5p was expressed in low quantities based on RT-qPCR. Our study demonstrates that miR-216a-5p plays an important role in SCLC cell growth and migration and modulates the $\mathrm{G} 2$ phase of the cell cycle.

$B c l-2$ was subsequently identified as a direct target gene of miR-216a-5p that is involved in SCLC pathogenesis under negative regulation by miR-216a-5p. In addition to its effect on Bcl-2, miR-216a-5p downregulation promoted the expression of the apoptosis-related genes Bax and Bad. Bcl-2, a wellknown apoptosis suppressor gene, is involved in the progression of multiple tumors. ${ }^{22,23}$ Studies have reported that many miRNAs can regulate the expression of $B c l-2 .{ }^{24}$ However, the mechanism through which $\mathrm{BCl}-2$ participates in SCLC biology under the regulation of miR-216a-5p remained obscure. Based on our previous study, $B c l-2$ was found to play a key role in the chemoresistance of SCLC due to its antiapoptotic function. ${ }^{18}$ The data in this study showed that $\mathrm{Bcl}-2$ promotes cell proliferation and tumorigenesis in nude mice by modulating the cell cycle. Furthermore, when we downregulated $\mathrm{Bcl}-2$ 
A

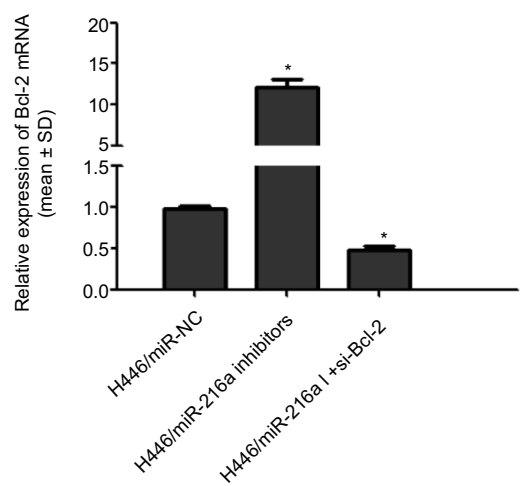

C

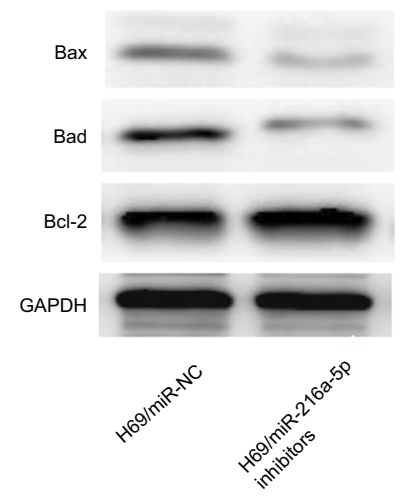

B

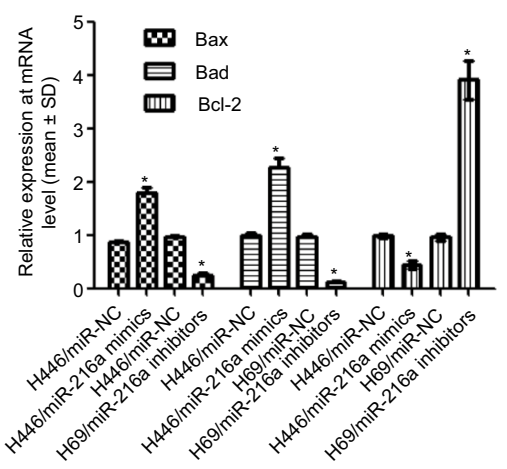

D

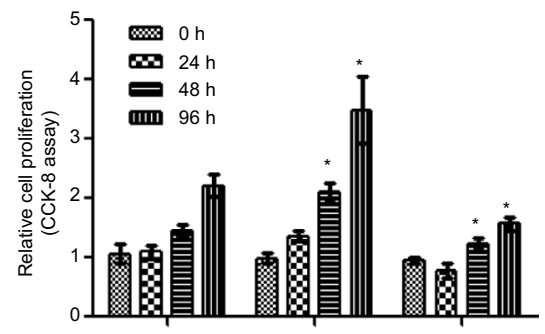

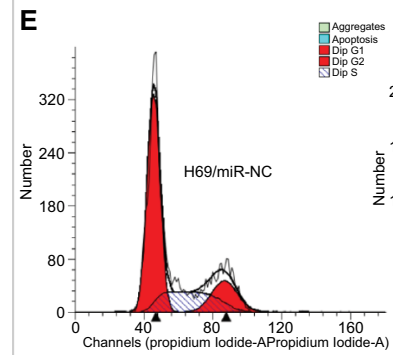

E

$\mathbf{F}$

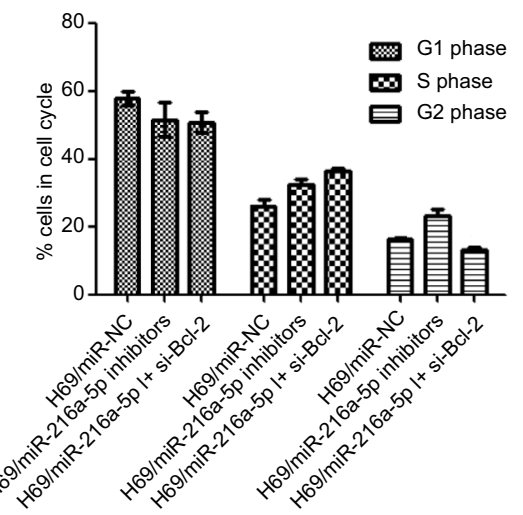

G

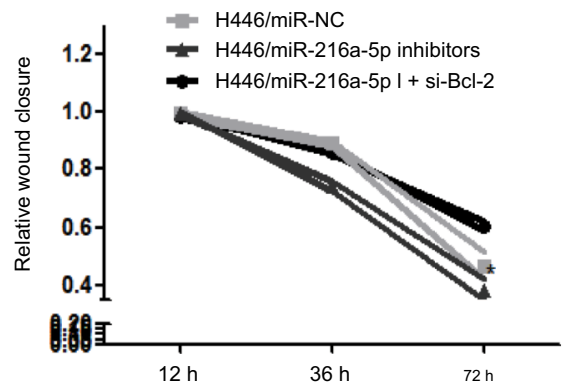

Figure $4 \mathrm{Bcl}-2$ mediates the effect of miR-216a-5p on SCLC pathogenesis.

Notes: (A) Rescue experiment of Bcl-2 downregulation by miR-216a-5p knockdown using RT-qPCR. (B) and (C) miR-216a-5p mediated expression of the apoptosis-related genes Bcl-2/Bad/Bax at the mRNA and protein levels. (D) miR-216a-5p decreased cell growth by targeting Bcl-2. (E) and (F) miR-216a-5p influenced the cell cycle of SCLC cells by targeting $\mathrm{Bcl}-2$. (G) The in vitro wound healing experiment shows that Bcl-2 knockdown rescued the effect of miR-2/6a-5p downregulation on SCLC cell migration. $* P<0.05$.

Abbreviations: SCLC, small cell lung cancer; RT-qPCR, real-time quantitative PCR; NC, negative control; CCK-8, Cell Counting Kit-8. 
expression by cotransfection with $B c l-2$ siRNA in a stable cell line in which miR-216a-5p was downregulated, the upregulation of $B c l-2$ was reversed and cell proliferation was inhibited, which is consistent with the effect of miR-216a-5p upregulation on cell proliferation. These results further suggest that miR-216a-5p might regulate SCLC cell proliferation through mediation of $\mathrm{Bcl}-2$ expression. Taken together, the results of this study indicate that miR-216a-5p may affect SCLC biology by activating the apoptosis signaling pathway, although more in-depth study will be needed.

Numerous studies have closely linked miR-216a-5p to a variety of human tumors. For example, miR-216a-5p was reported to be downregulated by more than 200 -fold in pancreatic ductal adenocarcinoma samples compared to that in normal pancreatic tissues, while the same situation was not observed in chronic pancreatitis samples. Similarly, upon transformation of pancreatic stellate cells to myofibroblast-like cells, ${ }^{18}$ a key step in pancreatic cancer progression, miR-216a-5p was downregulated. Moreover, the downregulation of miR-216a-5p was associated with pancreatic ductal adenocarcinoma cell migration, invasion, and epithelial-to-mesenchymal transition and pancreatic cancer progression. On the basis of those studies, our findings confirm that miR-216a-5p is a tumor suppressor.

In this study, we first validated our previous TaqMan miRNA microarray results by RT-qPCR and chose miR-216a-5p as the subject of our investigation of SCLC. Second, on the basis of up- and downregulation of miR-216a-5p expression through the use of miR-216a-5p mimics and inhibitors, respectively, we found in in vitro and in vivo experiments that miR-216a-5p inhibited cell proliferation, cell migration, and xenograft formation and influenced cell cycle status, particularly in the G2 phase. Finally, using bioinformatics and a luciferase reporter assay, we confirmed that miR-216a-5p targets the $\mathrm{Bcl}-2$ gene. $\mathrm{Bcl}-2$ promoted cell proliferation and migration and impacted the $\mathrm{G} 2$ phase of the cell cycle under regulation by miR-216a-5p. Furthermore, suppression of miR-216a-5p inhibited the expression of Bax and Bad while increasing $\mathrm{Bcl}-2$ expression. Our study suggests that miR-216a-5p is involved in SCLC pathogenesis via modulation of $\mathrm{Bcl}-2 / \mathrm{Bax} / \mathrm{Bad}$.

Moreover, our study demonstrated that the suppression of proliferation capacity of $\mathrm{H} 69$ cells by $\mathrm{Bcl}-2$ gene silencing might be due to a reduction of cells in the G2 phase of the cell cycle. Since the $\mathrm{S}$ phase does not seem to be impacted by Bcl-2 siRNA and small molecule inhibitors (eg, venetoclax) of Bcl-2 impact SCLC cell line viability, ${ }^{25}$ how do Bcl-2 siRNA or Bcl-2 inhibitors impact SCLC cells' survival in the context of miR-216a expression will be our next area of interest.

\section{Conclusion}

We have demonstrated that miR-216a-5p plays an important role in the pathogenesis of SCLC. miR-216a-5p expression significantly decreased cell proliferation and migration in SCLC through mediating the expression of $\mathrm{Bcl}-2 / \mathrm{Bax} / \mathrm{Bad}$ proteins. Based on these findings, we propose that miR216a-5p may be used as a therapeutic agent for SCLC.

\section{Acknowledgment}

This study was supported by the National Natural Science Foundation of China (81372508, 81702285), the Guangdong Natural Science Foundation (Special Fund for Scientific and Technological Development; 2017A030313644), the Guangdong Medical Research Foundation (A2017330), the fund for Innovative Experiments for College Students in Guangdong Medical University (ZYDM021), and the College Students' Innovation and Entrepreneurship Training Program (GDMU2017133).

\section{Disclosure}

The authors report no conflicts of interest in this work.

\section{References}

1. du L, Schageman JJ, Irnov, et al. MicroRNA expression distinguishes SCLC from NSCLC lung tumor cells and suggests a possible pathological relationship between SCLCs and NSCLCs. J Exp Clin Cancer Res. 2010;29(1):75.

2. Verma V, Choi JI. Simone CB 2nd. Proton therapy for small cell lung cancer. Transl Lung Cancer Res. 2018;7(2):134-140.

3. Taucher V, Mangge H, Haybaeck J. Non-coding RNAs in pancreatic cancer: challenges and opportunities for clinical application. Cell Oncol. 2016;39(4):295-318.

4. Mizuno K, Mataki H, Arai T, et al. The microRNA expression signature of small cell lung cancer: tumor suppressors of miR-27a-5p and miR-34b-3p and their targeted oncogenes. J Hum Genet. 2017;62(7): 671-678.

5. Liu HY, Chen J. Polymorphisms in miRNA binding site: new insight into small cell lung cancer susceptibility. Acta Pharmacol Sin. 2011;32(10):1191-1192.

6. Xiong $\mathrm{F}, \mathrm{Wu} \mathrm{C}$, Chang J, et al. Genetic variation in an miRNA-1827 binding site in MYCL1 alters susceptibility to small-cell lung cancer. Cancer Res. 2011;71(15):5175-5181.

7. Guo L, Liu Y, Bai Y, et al. Gene expression profiling of drug-resistant small cell lung cancer cells by combining microRNA and cDNA expression analysis. Eur J Cancer. 2010;46(9):1692-1702.

8. Roa W, Brunet B, Guo L, et al. Identification of a new microRNA expression profile as a potential cancer screening tool. Clin Invest Med. 2010;33(2):124.

9. Hou B, Jian Z, Chen S, et al. Expression of miR-216a in pancreatic cancer and its clinical significance. Nan Fang Yi Ke Da Xue Xue Bao. 2012;32(11):1628-1631. 
10. Zhang D, Zhao L, Shen Q, et al. Down-regulation of KIAA1199/CEMIP by miR-216a suppresses tumor invasion and metastasis in colorectal cancer. Int J Cancer. 2017;140(10):2298-2309.

11. Wang D, Li Y, Zhang C, Li X, Yu J. MiR-216a-3p inhibits colorectal cancer cell proliferation through direct targeting COX-2 and ALOX5. $J$ Cell Biochem. 2018;119(2):1755-1766.

12. WuY, Zhang J, Zheng Y, Ma C, Liu XE, Sun X. miR-216a-3p Inhibits the Proliferation, Migration, and Invasion of Human Gastric Cancer Cells

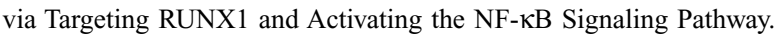
Oncol Res. 2018;26(1):157-171.

13. Li H, Li T, Fan J, et al. miR-216a rescues dexamethasone suppression of osteogenesis, promotes osteoblast differentiation and enhances bone formation, by regulating c-Cbl-mediated PI3K/AKT pathway. Cell Death Differ. 2015;22(12):1935-1945.

14. Su Y, Bai M, Zhu LP, et al. Pirh2 shRNA mediated by psiRNA-hH1 vector plasmid effectively inhibits the proliferation of lung carcinoma cells: in vitro and in vivo experiments. Zhonghua Yi Xue Za Zhi. 2007;87(17):1199-1203.

15. Zhou H, Zheng L, Lu K, et al. Downregulation of Cohesin Loading Factor Nipped-B-Like Protein (NIPBL) Induces Cell Cycle Arrest, Apoptosis, and Autophagy of Breast Cancer Cell Lines. Medical Science Monitor. 2007;23:4817-4825.

16. Sun Y, Zeng C, Gan S, et al. LncRNA HOTTIP-Mediated HOXA11 Expression Promotes Cell Growth, Migration and Inhibits Cell Apoptosis in Breast Cancer. Int J Mol Sci. 2018;19(2):472.

17. Ye M, Wei T, Wang Q, et al. TSPAN12 promotes chemoresistance and proliferation of SCLC under the regulation of miR-495. Biochem Biophys Res Commun. 2017;486(2):349-356.
18. Sun Y, Hu B, Wang Q, et al. Long non-coding RNA HOTTIP promotes BCL-2 expression and induces chemoresistance in small cell lung cancer by sponging miR-216a. Cell Death Dis. 2018;9(2):85.

19. Bai Y, Wang YL, Yao WJ, et al. Expression of miR-32 in human nonsmall cell lung cancer and its correlation with tumor progression and patient survival. Int J Clin Exp Pathol. 2015;8(1):824-829.

20. Li XX, Li RJ, Zhao LJ, Liu NB, Wang P. Expression of molecular factors correlated with metastasis in small cell lung cancer and their significance. Int J Clin Exp Pathol. 2015;8(11):14676-14684.

21. Pak MG, Lee CH, Lee WJ, Shin DH, Roh MS. Unique microRNAs in lung adenocarcinoma groups according to major TKI sensitive EGFR mutation status. Diagn Pathol. 2015;10:99.

22. Torrealba N, Rodriguez-Berriguete G, Fraile B, et al. PI3K pathway and Bcl-2 family. Clinicopathological features in prostate cancer. Aging Male. 2018:1-12.

23. Reed JC. Bcl-2 on the brink of breakthroughs in cancer treatment. Cell Death Differ. 2018;25(1):3-6.

24. Sun XX, Zhang SS, Dai CY, et al. LukS-PV-Regulated MicroRNA125a-3p Promotes THP-1 Macrophages Differentiation and Apoptosis by Down-Regulating NF1 and Bcl-2. Cell Physiol Biochem. 2017;44(3):1093-1105.

25. Lochmann TL, Floros KV, Naseri M, et al. Venetoclax is effective in small-cell lung cancer with high BCL-2 expression. Clin Cancer Res. 2018;24(2):360-369. 


\section{Supplementary materials}

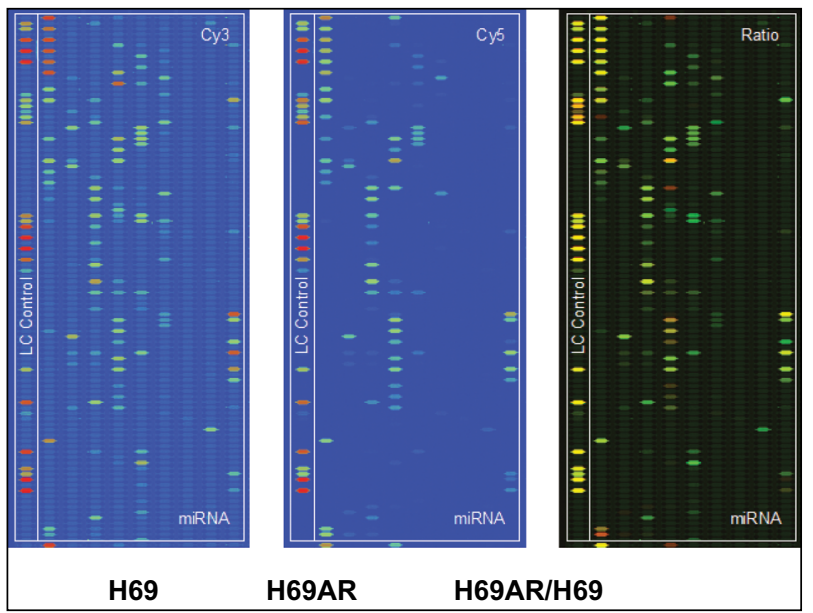

Figure SI miRNA profiles from Cy3 and Cy5 images of $\mathrm{H} 69 \mathrm{AR} / \mathrm{H} 69$ cells by TaqMan miRNA microarray analysis.

Abbreviation: miRNA, microRNA.

\section{Primers}

miR-216a-5pRTprimer(5'-3'): CTCAACTGGTGTCGTGGA miR-216a-5p forward primer $\left(5^{\prime}-3^{\prime}\right)$ : TGTCGCAAATCT CTGCAGG

miR-216a-5p reverse primer $\left(5^{\prime}-3^{\prime}\right)$ : CAGAGCAGGGTC CGAGGTA

Bcl-2 forward primer $\left(5^{\prime}-3^{\prime}\right)$ : GAACTGGGGGAGG ATTGTGG

Bcl-2 reverse primer (5'-3'): CCGTACAGTTCCACAAAGGC
Bax forward primer $\left(5^{\prime}-3^{\prime}\right)$ : CCCGAGAGGTCTTTTTCCGAG Bax reverse primer $\left(5^{\prime}-3^{\prime}\right)$ : CCAGCCCATGATGGTTCTGAT Bad forward primer $\left(5^{\prime}-3^{\prime}\right)$ : CCCAGAGTTTGAGCCGAGTG Bad reverse primer $\left(5^{\prime}-3^{\prime}\right)$ : CCCATCCCTTCGTCGTCCT GAPDH forward primer (5'-3'): GGGCTGCTTTTAACTCTG GAPDH reverse primer $\left(5^{\prime}-3^{\prime}\right)$ : TGGCAGGTTTTT CTAGACGG

\section{miR-2I6a-5p mimics and inhibitor sequences}

Hsa-miR-216a-5p mimic: UAAUCUCAGCUGGCAACUGUGAACAGUUGCCAGCUGAGAUUAUU Hsa-miR-216a-5p inhibitor: UCACAGUUGCCAGCUGA GAUUA

\section{Antibodies information}

Rabbit antihuman monoclonal antibody Bcl-2 (\#5284; Santa Cruz Biotechnology, Santa Cruz, CA, USA)

Rabbit antihuman monoclonal antibody Bad (\#9239; Santa Cruz Biotechnology)

Rabbit antihuman monoclonal antibody Bax (\#2772; Santa Cruz Biotechnology)

The stable overexpression or silence miR-216a-5p expression cell lines and control cell lines were constructed by lentivirus infection, which were purchased from GenePharma (Shanghai, China). Cell transfection was carried out using Lipofectamine 2000 (Thermo Fisher Scientific, Waltham, MA, USA) following the manufacturer's protocol.
Cancer Management and Research

\section{Publish your work in this journal}

Cancer Management and Research is an international, peer-reviewed open access journal focusing on cancer research and the optimal use of preventative and integrated treatment interventions to achieve improved outcomes, enhanced survival and quality of life for the cancer patient. The manuscript management system is completely online and includes

\section{Dovepress}

a very quick and fair peer-review system, which is all easy to use. Visit http://www.dovepress.com/testimonials.php to read real quotes from published authors. 